\title{
Interferometric SAR Signal Analysis in the Presence of Squint
}

\author{
Marc Bara, Student Member, IEEE, Rolf Scheiber, Antoni Broquetas, Member, IEEE, and \\ Alberto Moreira, Senior Member, IEEE
}

\begin{abstract}
This paper develops an analysis of the SAR impulse response function from the interferometric point of view, with the intention of studying its phase behavior in the presence of high squint angle values. It will be pointed out that in this case, a phase ramp is present in the range direction, which, in combination with a certain degree of misregistration between the two images induces an offset in the generated interferometric phase. This behavior, if not compensated, imposes strong limits on the performance of the interferometric techniques in a squinted case, especially for airborne SAR systems. The article proposes two new techniques, which are appropriate to correct the phase bias coming from this source. The first one is based on a modification of the azimuth compression filter, which cancels the phase ramp of the range impulse response function for one specific squint value. In case the SAR processing is performed with variable squint over range, we propose a second method oriented to estimating the expected misregistration and thus, the phase bias by means of an iterative approach. Simulated data as well as real corner reflector responses are used to show that the correct topography can be recovered precisely even in the presence of phase bias coming from the squinted geometry.
\end{abstract}

Index Terms-Digital elevation model, interferometry, remote sensing, synthetic aperture radar (SAR).

\section{INTRODUCTION}

$\mathbf{S}_{\mathrm{n}}$ YNTHETIC aperture radar (SAR) has been used for many years for the generation of two-dimensional (2-D) reflectivity images of the Earth's surface [1]. The generation of such a product requires the coherent combination of the echoes recorded by the SAR sensor in order to properly focus all the targets within the image. Many algorithms, which carry out this task in an efficient way, have been developed in the last decades, including range-Doppler processing approaches, wavenumber techniques, or chirp scaling-based algorithms [2]-[6]. All of them must cope with the presence of the so-called squint angle, which is directly associated with the center value of the Doppler variation, i.e., the Doppler centroid. It is generally present as a result of attitude changes of the SAR sensor, which are more common in the airborne case since the radar is mounted on a platform that experiences rotations and

Manuscript received September 16, 1999; revised March 31, 2000. This work was supported by the Spanish Commission for Science and Technology (CICYT) ref. TIC99-1050-C03-01, and in part by the Catalan Commission for Research (CIRIT).

M. Bara is with the Department of Signal Theory and Communications, Polytechnic University of Catalonia, E-08034 Barcelona, Spain (e-mail: mabara@tsc.upc.es).

R. Scheiber and A. Moreira are with the Institut für Hochfrequenztechnik und Radarsysteme, Deutsches Zentrum für Luft- und Raumfahrt e.V., D-82230 Oberpfaffenhofen, Germany (e-mail: Rolf.Scheiber@dlr.de).

A. Broquetas is with the Institute of Geomatics, E-08038 Barcelona, Spain. Publisher Item Identifier S 0196-2892(00)08899-9. motion errors as a result of atmospheric turbulences, strong wind conditions, etc. In the spaceborne case, the trajectories are much more stable, but the Doppler centroid exists basically as a consequence of the Earth's rotation and curvature. In the case of ERS, the satellite is steered to compensate the Doppler centroid [7]. For RADARSAT [8], high squint values occur due to the reduced steering of the satellite's attitude.

In both air- and spaceborne cases, it is of extreme importance to consider the correct properties of the squinted SAR signal [9] to avoid defocusing. Appropriate techniques as high order interpolations or high order filters, as described in [10] for the chirp scaling algorithm, must be employed.

One important application of SAR is based on the combination of 2-D images acquired from two different points of view in an across-track configuration in order to form an interferometer capable of retrieving topographic information, i.e., digital elevation models (DEM) [11]. SAR interferometric techniques are based on the phase information, i.e., every pixel of the calculated interferogram must contain the phase difference between the imaged point and the two antennas. This is only possible after an accurate coregistration process, which should be able to match all the pixels of one image to the other with very high precision. The coregistration step increases the quality of the interferogram, easing in this way the phase unwrapping procedure [12] and improving the accuracy of the height values in the finalDEM. Several approaches have been proposed, based on conventional blockmatching methods to maximize the cross-correlation measurement, or making use of the combination of different spectral contents as proposed recently in [13]. In any case, the accuracy of the coregistration methods is never infinite when no DEM is known a priori, since the misregistration between both images is always a function of the topography, the parameter to be estimated.

In this context, this paper studies the applicability of SAR images focused with phase preserving algorithms adapted to high squint to generate high quality interferograms for mapping applications. The effect of the squint angle is considered from the interferometric point of view, i.e., its impact on the phase values obtained after the coregistration process is assessed. The objective of the paper is first to derive the signal properties in high squinted geometries, without small angle approximations. In this way, the presence of some limitations in the retrieval of the correct interferometric phase values is shown. The paper also presents two new methods to overcome this problem, extending in this way the applicability of SAR interferometry for high squint values.

In Section II, the characteristics of the squinted SAR signal are evaluated, in particular, its phase behavior in a general case. 
The expressions are also extended to the airborne case, where a term coming from the motion compensation step is added. The final expression is validated by means of real interferometric SAR data acquired with the E-SAR system [14] of DLR. In Section III, the paper deals with the interferometric problem, considering the effects of the squint in combination with a certain degree of misregistration, which leads to the presence of a noticeable interferometric phase bias. In Appendix B, the results are also confirmed when using a model of distributed scatterers instead of point targets, finding the consequences on the coherence as well. Section IV presents two methods developed with the intention of increasing the phase reliability in the squinted case. The first one is based on a modification of one of the filters applied during SAR processing, whereas the second method carries out an estimation of the misregistration starting from the initial phase values and increasing its estimated accuracy in an iterative way. Appendix $\mathrm{C}$ studies the convergence of these iterations. The end of Section IV shows the results of evaluating these two approaches with simulated point targets and with real interferometric SAR data. Finally, Section V concludes the paper.

\section{SAR IMPULSE RESPONSE CHARACTERISTICS IN A SQUINTED GEOMETRY}

It is well known that the impulse response function (IRF) of a narrowband SAR system can be considered as a two dimensional weighted sinc function, obtained after the process of compression of the raw data coming from an individual target [1]. Specifically, the shape of this response is dependent on the antenna diagram, the envelope of the transmitted pulse and the weighting functions applied during the processing step in both coordinates, range, and azimuth [2]. This section presents a review of the signal formulation, oriented to derive explicitly the impact of the squint angle on its phase. It will be pointed out that, in the case of chirp scaling [5] or range-Doppler algorithms [6], spectral shifts are introduced by the variation with range of the azimuth compression function. From the wavenumber point of view [4], they appear as a result of the projection of the transmitted frequency onto both wavenumber axes. For the airborne case, the additional effects due to motion compensation will also be discussed.

\section{A. Signal Expression: Phase Behavior in the Presence of Squint}

We start from the signal expression in the so called rangeDoppler domain [5], [15] prior to azimuth compression. It is assumed that proper range compression (including secondary range compression), range cell migration correction and motion compensation (for the airborne case) have been already carried out at this stage. For a target located at a minimum range distance $r_{0}$ (closest approach distance) the signal takes the form:

$$
\begin{aligned}
S\left(\tau, f_{a}\right)= & C_{0} \cdot s_{r}\left(\tau-\tau_{0}\right) \cdot w_{a}\left(\frac{f_{a}-f_{c}}{f_{r}}\right) \cdot \exp \left[-j 2 \pi f_{a} t_{0}\right] \\
& \cdot \exp \left[-j \frac{4 \pi}{\lambda} r_{0} \sqrt{1-\left(\frac{\lambda f_{a}}{2 v}\right)^{2}}\right]
\end{aligned}
$$

where

$\tau \quad$ range time variable;

$f_{a} \quad$ azimuth frequency;

$f_{r} \quad$ first derivative of the Doppler frequency within the synthetic aperture (Doppler rate);

$f_{c} \quad$ Doppler centroid;

$t_{0} \quad$ azimuth time related to the position of the target;

$\lambda \quad$ wavelength value;

$v \quad$ effective platform forward velocity, which includes the effect of Earth's curvature and rotation for the spaceborne case [16].

The functions named $s_{r}$ and $w_{a}$ are the envelopes in range time and azimuth frequency domains, respectively, while $C_{0}$ is an arbitrary complex constant. In particular, the frequency $f_{a}$ takes values in the interval

$$
f_{c}-B_{a z} / 2 \leq f_{a} \leq f_{c}+B_{a z} / 2
$$

where $B_{a z}$ is defined as the azimuth bandwidth. The range position of the impulse response, given by $\tau_{0}$, is expressed in terms of time as

$$
\tau_{0}=\left(2 \cdot r_{0}\right) / c
$$

For simplification, we use the following relationship [17] in the next expressions

$$
\cos ^{2} \beta\left(r_{0}\right)=1-\left(\frac{\lambda f_{c}\left(r_{0}\right)}{2 v}\right)^{2}
$$

which relates the squint angle $\beta$ to the Doppler centroid frequency $f_{c}$. The possible dependence on the range target position has been included explicitly.

Usually, the last step of the processing with a range-Doppler or chirp scaling processor consists of the multiplication with the azimuth-compression filter

$$
H_{a z}\left(\tau, f_{a}\right)=\exp \left[-j \frac{2 \pi}{\lambda} c \tau \cdot\left(1-\sqrt{1-\left(\frac{\lambda f_{a}}{2 v}\right)^{2}}\right)\right]
$$

which is tuned to every range bin in order to focus all the responses along the range dimension. This is obtained through the variation of $\tau$ in (5). The operation will eliminate the hyperbolic phase evolution of the range-compressed signal in the azimuth frequency domain, but only for the range time position equal to $\tau_{0}$. For other $\tau$, even within the main lobe of the response, there will remain a residual term with the shape of the square root of (5). In Appendix A, we detail a Taylor expansion of this term around the Doppler centroid frequency [with respect to $\left(f_{a}-f_{c}\right)$ ], in order to identify the contribution of each coefficient. It is shown that the constant term of the expansion is responsible for a phase ramp, since it is indeed dependent on $\tau$. On the other hand, the linear coefficient induces a signal shift (also dependent on $\tau$ ) when transformed back to the azimuth time domain. Finally, the second order factor is shown to be negligible within the main lobe of the response, where $\tau-\tau_{0}$ is smaller than the resolution cell.

With these considerations, it is possible to find a compact expression for the SAR impulse response, which includes phase 
ramps in range and azimuth due to the presence of squint. It is obtained by transforming the compressed signal to the azimuth time domain

$$
\begin{aligned}
s_{c}(\tau, t)= & C \cdot s_{r}\left(\tau-\tau_{0}\right) \\
& \cdot s_{a}\left(t-t_{0}-\frac{c}{2} \cdot \frac{\tan \beta\left(r_{0}\right)}{v} \cdot\left(\tau-\tau_{0}\right)\right) \\
& \cdot \exp \left[-j \frac{4 \pi}{\lambda} r_{0}\right] \cdot \exp \left[j 2 \pi f_{c}\left(t-t_{0}\right)\right] \\
& \cdot \exp \left[-j 2 \pi f_{0} \cdot\left(1-\cos \beta\left(r_{0}\right)\right) \cdot\left(\tau-\tau_{0}\right)\right]
\end{aligned}
$$

where

$$
\begin{array}{ll}
\text { subindex } c & \text { "compressed"; } \\
f_{0} & \text { radar central frequency; } \\
C & \text { complex constant; } \\
s_{a} & \text { inverse transform of } w_{a} .
\end{array}
$$

The displacement of the sidelobes of $s_{a}$ is characteristic for the so-called zero-Doppler geometry, where every scatterer is located at the time and distance corresponding to the minimum approach position, even if the squint is not null. In this case, the phase at the peak corresponds to $r_{0}$ as well. It is possible to obtain an output in a beam-centered or squinted geometry by adding a certain range varying linear phase to the filter described by (5). Anyway, compression to beam center is not usually done in practice, and hence, the analysis presented here is based on compression in zero-Doppler geometry.

Equation (6) gives an accurate description of the phase behavior in a squinted case. Specifically, the range phase ramp is of extreme importance for interferometry, since any possible range misalignment between the two peaks yields a phase bias (see Section III). It is worth noting that the ramp can be understood as a spectral shift, which has been introduced by the azimuth compression filter. Its expression is

$$
f_{\tau 0}=-f_{0} \cdot\left(1-\cos \beta\left(r_{0}\right)\right) \text {. }
$$

This shift was identified also in the wavenumber formulation, as reported in [4]. In that case the so called Stolt interpolation is carried out to prepare the data for efficient azimuth focusing. The process is based on a change of variables between the transmitted frequency and the radial frequency. In squint mode processing, the transmitted center frequency is projected onto two orthogonal axes after image formation. Thus, (7) can be interpreted as the shift induced by the projection onto the $f_{\tau}$ axis of the point $f_{0}$ located on the $f$ axis, which is rotated by an angle equal to $\beta$ from boresight. For narrowband systems, this shift can be considered to be constant over the transmitted bandwidth.

This result is important for interferometric applications, since this spectral shift can impose severe requirements on the interferometric processing chain, as will be shown in Section III. In Appendix B, we study the consequences of (7) on the first and second order statistics of the interferometric phase.

\section{B. Effects of Motion Compensation in the Airborne Case}

Section II-A pointed out the appearance of a range spectral shift introduced by the squint angle during azimuth compression. However, in order to obtain an expression general enough to comprise all the kinds of SAR platforms, space- and airborne, another effect must be taken into account. In particular, it is well known that in the airborne case, it is very important to compensate the trajectory instabilities of the SAR sensor during the focusing step [18], in the so-called motion compensation (MoCo) step. Otherwise, the quality of the final image degrades seriously [19]. In [20], several MoCo options from the interferometric point of view were analyzed.

Basically, during the MoCo procedure, the data must be multiplied by an exponential phase term in order to correctly compensate for the deviations between the nominal and the actual trajectories [17]. This factor depends on the incidence angle from the radar to the target and therefore, on the slant-range axis $r$ as described by [21]. As the slant range varies along the swath, the extended chirp scaling (ECS) algorithm [15] proposes a two-step scheme. First, a coarse compensation is applied for the center of the swath. Next, after range compression and range cell migration correction (RCMC), it is possible to multiply the signal with a range-varying factor, oriented to carry out the so-called second order motion compensation to allow a proper range compression. Indeed, the fact that the exact MoCo is performed after RCMC makes this configuration appropriate for high-squinted interferometric data, where the migration is important in terms of range bins. In this case, the signal represented by (6) can be focused without phase distortions. Otherwise, its characteristics would degrade when using other options which apply motion compensation to the range-migrated data [20], [21].

Either using a MoCo procedure with one or two steps, at a specific stage, the signal is multiplied by a factor of the form

$$
H_{\mathrm{MoCo}}(t, r)=\exp \left[j \frac{4 \pi}{\lambda} \Delta d(t, r)\right]
$$

where

$t$ azimuth time,

$r$ range axis;

$\Delta d \quad$ distance to be compensated (i.e. the difference between the nominal and actual range to the target), which is variable in both dimensions, azimuth and slant range.

Any linear component of $\Delta d$ along range leads to a spectral shift in the final impulse response and consequently, a phase ramp in the time domain. This shift is approximated by the first derivative of $\Delta d$ at the range target position $\tau_{0}$

$$
f_{\tau 0}(t, r)=f_{0} \cdot \frac{\partial \Delta d(t, r)}{\partial r} .
$$

For the center of aperture position located at $t=t_{c}$, and considering that the variation of the shift in (9) within the synthetic aperture is small, then the spectral shift after azimuth compression can be approximated as

$$
f_{\tau 0}\left(t_{c}, r\right) \cong f_{0} \cdot \frac{\partial \Delta d\left(t_{c}, r\right)}{\partial r}
$$

Equation (10) can be used in conjunction with (7) in order to include the contribution of both factors, MoCo and squint, on 
the amount of spectral shift. Then it is possible to derive a new expression for the focused SAR impulse response function

$$
\begin{aligned}
s_{c}(\tau, t)= & C \cdot s_{r}\left(\tau-\tau_{0}\right) \\
& \cdot s_{a}\left(t-t_{0}-\frac{c}{2} \cdot \frac{\tan \beta\left(r_{0}\right)}{v} \cdot\left(\tau-\tau_{0}\right)\right) \\
& \cdot \exp \left[-j \frac{4 \pi}{\lambda} r_{0}\right] \cdot \exp \left[j 2 \pi f_{c}\left(t-t_{0}\right)\right] \\
& \cdot \exp \left[-j 2 \pi f_{0} \cdot\left(1-\cos \beta\left(r_{0}\right)\right.\right. \\
& \left.\left.\quad-\left.\frac{\partial \Delta d\left(t_{c}, r\right)}{\partial r}\right|_{r=r_{0}}\right) \cdot\left(\tau-\tau_{0}\right)\right]
\end{aligned}
$$

which is appropriate not only for satellite sensors but also for airborne SAR data.

\section{Verification with Real Point Targets}

We have processed SAR data acquired by the E-SAR system of the DLR [14] over the test site of Oberpfaffenhofen, Germany, in 1998. The system characteristics for the flight, as well as the processing parameters, are shown in Table I. This data set contains the response of five corner reflectors of different sizes (see Fig. 1) for calibration purposes. They are positioned approximately in the same azimuth position, whereas their slantrange distance to the master antenna varies from 4295 to 4739 $\mathrm{m}$.

During the flight, the aircraft experienced horizontal and vertical deviations in the range of $\pm 10 \mathrm{~m}$ and $\pm 2 \mathrm{~m}$, respectively. These values were taken into account during the motion compensation step and led to the range-varying phase ramps displayed in Fig. 2. A Hamming weighting of 0.54 was used for range side-lobe suppression. The average squint angle for this flight is $2.7^{\circ}$ and was assumed constant over range during processing. Thus, the total spectral shift led to a phase ramp of around $19^{\circ} 1 / \mathrm{m}$ in the area of the corner reflectors (Table III, second column), according to the theoretical expressions. These values have been checked after the processing of the data, and the range cuts for the five responses are shown in Fig. 3. As we can see, all the targets present a phase ramp within the main lobe of the response, and the measured slope equals the predicted one of (11).

\section{EFFECTS OF SQUINT AND MOCO TERMS ON INTERFEROMETRY}

As commented in Section II, the presence of the squint angle induces several effects on the SAR impulse response function. The next concern is to find out the consequences regarding the computation of the targets position by means of an interferometric process, which consists of the crossmultiplication of two interferometric SAR images acquired from slightly different positions [11]. It will be shown that small misregistration errors have a strong influence on the final precision of the digital elevation model (DEM) in case of squint, not only because of a degradation of the coherence [22], but also due to the appearance of a phase bias.
TABLE I

DATA CHARACTERISTICS FOR THE TEST SITE OF OBERPFAFFENHOFEN (E-SAR SYSTEM)

\begin{tabular}{cc}
\hline Parameter & Value \\
\hline Wavelength & $0.031219557 \mathrm{~m}$ \\
Bandwidth & $100 \mathrm{MHz}$ \\
Chirp Duration & $5 \mu \mathrm{s}$ \\
Sampling Rate & $100 \mathrm{MHz}$ \\
PRF & $1000 \mathrm{~Hz}$ \\
Forward Velocity & $82.23 \mathrm{~m} / \mathrm{s}$ \\
Flight Altitude & $3065 \mathrm{~m}$ (above ground) \\
Track Center & $11.26^{\circ}$ Longitude \\
Coordinates & $48.06^{\circ} \mathrm{Latitude}$ \\
Presumming Factor & 4 \\
Slant Range Resolution & $3 \mathrm{~m}$ \\
Azimuth Resolution & $0.5 \mathrm{~m}$ \\
SLC pixel spacing in & $1.498 \mathrm{~m}$ \\
range & \\
SLC pixel spacing in & $0.33 \mathrm{~m}$ \\
azimuth & \\
Squint Angle & $2.7^{\circ}$ \\
Effective baseline & $1.5 \mathrm{~m}$ \\
\hline
\end{tabular}

\section{A. Interferometric Phase Bias}

During the interferometric process, the signals coming from the two channels are combined in order to obtain the interferometric phase information for every ground resolution cell. It is well known that, in this case, every pixel of the second image (slave) must correspond very precisely to the pixel in the first image (master) from the same cell on the observed surface [23]-[25]. The coregistration procedure, however, is not able to achieve an infinite precision, since the misalignment between both channels comes from the terrain relief itself. It is normally established that the accuracy in the coregistration step must accomplish a threshold in the order of $1 / 10$ of the resolution cell, which would cause a negligible statistical decorrelation [24]. However, it was shown that for squinted data and also in the case of ScanSAR interferometry the requirements on azimuth coregistration accuracy are much higher [13]. In this paper, we show that the coregistration accuracy in range is also critical in the case of considerable squint and/or terrain topography. As a result of the phase ramp within the main lobe of the target impulse response, even a small degree of misregistration is enough to introduce an important phase bias. In Fig. 4, this effect is illustrated. Although the ramps are cancelled when multiplying one image with the conjugate of the second one, the small shift between them yields an offset in the final phase value with respect to the correct one. Note that the amount of misregistration is related to the unknown topography changes and cannot be considered be systematic. From (11), the interferometric phase results in

$$
\begin{aligned}
\phi_{\text {int }} & =-\frac{4 \pi}{\lambda}\left(r_{1}-r_{2}-\Delta r_{m i s} \cdot\left(1-\cos \beta-\left.\frac{\partial \Delta d}{\partial r}\right|_{r=r_{2}}\right)\right) \\
& =\phi_{\text {ideal }}+\frac{4 \pi}{\lambda} \Delta r_{m i s} \cdot\left(1-\cos \beta-\left.\frac{\partial \Delta d}{\partial r}\right|_{r=r_{2}}\right)
\end{aligned}
$$

where $\Delta r_{m i s}$ is the range misregistration calculated with the peak positions in image 1 minus image 2 , and the effects of the squint angle and the MoCo term are evaluated for the target 


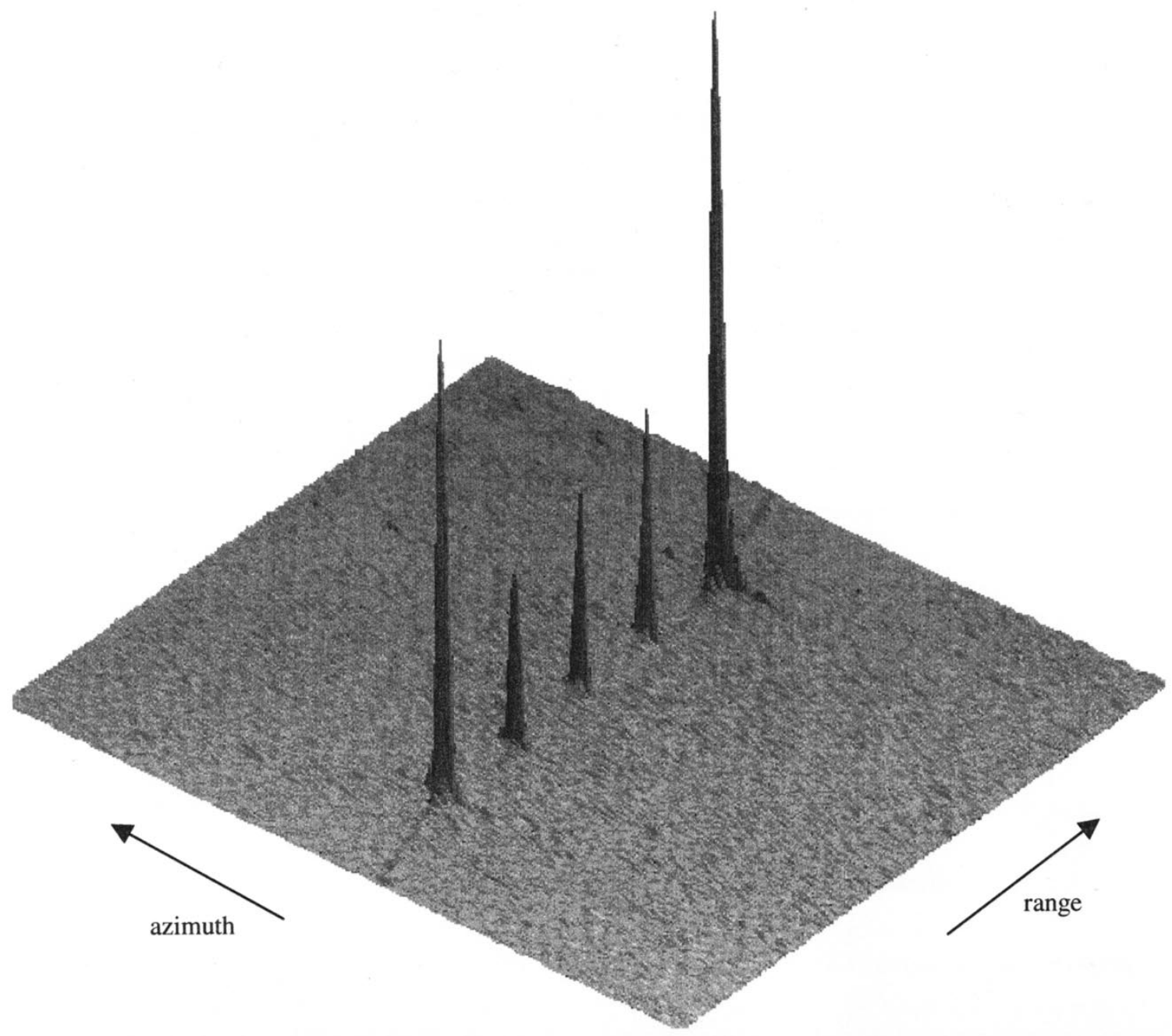

Fig. 1. Intensity of corner reflector responses as obtained by the E-SAR system for the Oberpfaffenhofen testsite.

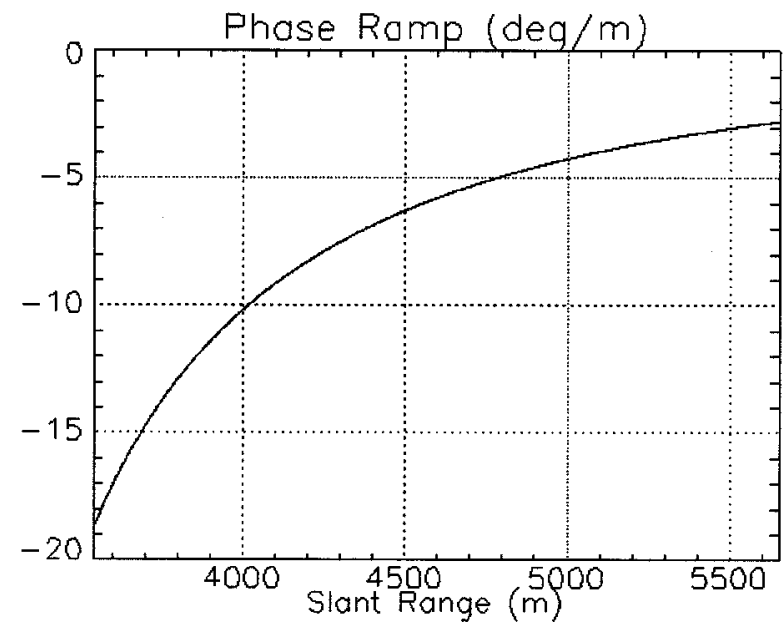

Fig. 2. Phase ramp within the range IRF due to the range update of the motion compensation phase function as a function of slant range position.

range position. For the interferogram generation, it was assumed that the azimuth filters of the two images were centered on the same Doppler frequency in order to avoid decorrelation. In this way, the bias becomes

$$
\phi_{\text {bias }}=\phi_{\text {int }}-\phi_{\text {ideal }}=\frac{4 \pi}{\lambda} \Delta r_{m i s} \cdot\left(1-\cos \beta(r)-\frac{\partial \Delta d}{\partial r}\right)
$$

where a general dependence on $r$ has been considered. Equation (13) is a general expression that can be applied to estimate the phase precision, given the expected misregistration and the squint angle for any airborne or spaceborne SAR platform. From this point of view, it is possible to define an effective squint angle containing the possible contribution of the MoCo term

$$
\beta_{e f}(r)=\arccos \left(\cos \beta(r)+\frac{\partial \Delta d}{\partial r}\right)
$$

leading to a more compact expression for the phase bias:

$$
\phi_{\text {bias }}=\frac{4 \pi}{\lambda} \Delta r_{m i s} \cdot\left(1-\cos \beta_{e f}(r)\right) .
$$

Equation (15) shows that the interferometric phase bias coming from this effect can be considerable in some situations, limiting 

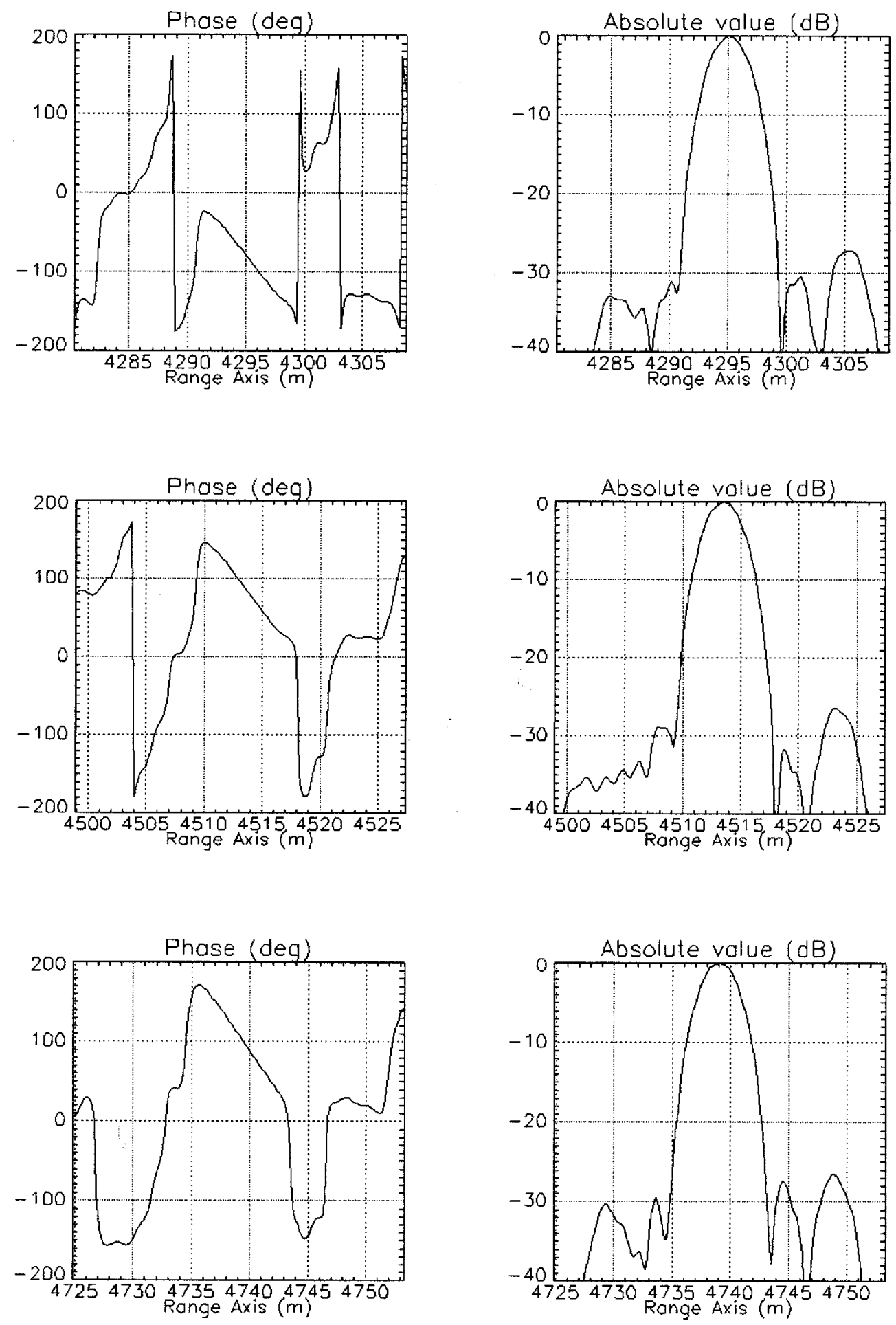

Fig. 3. IRF in range for the three corner reflectors focused with unmodified processor filter function. The near, middle, and far range corner reflectors from Fig. 1 have been selected for representation.

seriously the performance of the interferometric technique. In Appendix B, this investigation is confirmed also with distributed targets and the effects of squint on the estimated cross-correlation (coherence) are derived.

\section{B. Airborne Example}

For the parameters of the E-SAR system of DLR (Table I), $15^{\circ}$ of squint and a misregistration of $15 \mathrm{~cm}$ (corresponding to
$10 \%$ of a range sample), induced for example by topographic changes in the scene over range, the corresponding phase bias would reach $120^{\circ}$, approximately. If not corrected, this translates into height errors of 10-20 m depending on the radar look angle. Section IV of this paper presents two methods to correct for this error.

It is worth noting that in the azimuth direction, the phase ramp defined by the first exponential term of (6) is less critical from 


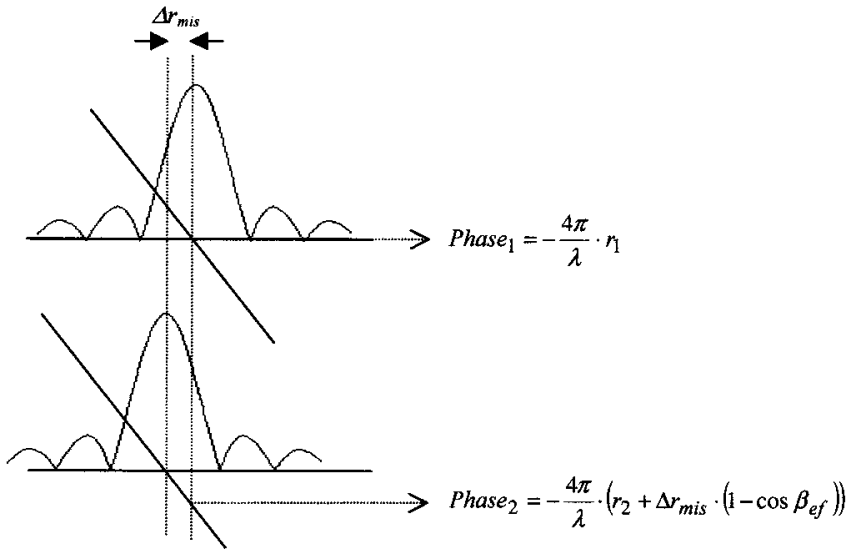

Fig. 4. Phase bias in the presence of misregistration and spectral shift.

the misregistration point of view, since it is considered that both images are correctly coregistered in this coordinate. This is usually the case of a single-pass interferometric system like E-SAR, where the positions of both antennas are known very precisely.

\section{Spaceborne Example}

In the spaceborne case, the effective squint is determined by the attitude of the spacecraft and the Earth's rotation and curvature, whereas the attitude of the spacecraft can be steered to eliminate the effect of rotation and curvature to a great extent [26]. Unfortunately, for the Canadian RADARSAT satellite, this cannot be performed and the effective squint can become relatively large [27]. In case interferometry is performed with a range misregistration of $1 \mathrm{~m}$ and $1^{\circ}$ of effective squint, a phase bias of $2^{\circ}$ occurs according to (15). For a baseline of $200 \mathrm{~m}$, this translates into a height error of less than $1 \mathrm{~m}$. Thus, the effect of the phase ramp in range is very small and can be neglected in this case. However, for an azimuth misregistration of $10 \%$ of the resolution cell, it can be shown that due to the range variation of the Doppler centroid imposed by a yaw angle of $3^{\circ}$ interferometric phase errors as large as $30^{\circ}$ occur from near to far range. This leads to height errors larger than $25 \mathrm{~m}$ in case of a baseline of $100 \mathrm{~m}$. To correct for this error, many tiepoints spread over range or a much higher coregistration accuracy is required in the azimuth direction. An efficient algorithm which assures this improved accuracy has been presented in [13].

\section{METhOdS FOR INTERFEROMETRIC PHASE BIAS COMPENSATION}

This section presents two different possibilities to decrease the effect of the phase bias given by (15). First, a modification of one phase function (the azimuth compression filter) used during the processing stage is proposed. Its main objective is to cancel the phase ramp, which exists in the SAR impulse response function (see Section II). The second option explores the idea of estimating the range misregistration between both SAR images, to cancel out the errors in the case of variable squint in range. The first method leads to a scaling of the interferometric phase, which must be accounted for in further evaluations, whereas the second method needs the exact knowledge of any prior coregistration operations applied to the data.

\section{A. Modification of the Azimuth-Compression Filter for Phase Ramp Cancellation}

In Section II, a general expression for the SAR impulse response function has been derived, which takes into account the phase ramps that exist in both dimensions of the data. As was mentioned, responsible for the range ramp is the azimuth-compression filter applied in the last step of the processing, as well as the motion compensation term in the airborne case. In order to prevent the appearance of this ramp, which has consequences even in cases of very small misregistration, a modified version of the filter of (5) including a factor defined as $\beta_{g}$ is proposed

$H_{a z}\left(\tau, f_{a}\right)=\exp \left[-j \frac{2 \pi}{\lambda} c \tau \cdot\left(\cos \beta_{g}-\sqrt{1-\left(\frac{\lambda f_{a}}{2 v}\right)^{2}}\right)\right]$.

This filter can be very useful to control the behavior of the phase ramp in range. Indeed, following the same considerations as in Section II, the expression of the impulse response of (11) changes to

$$
\begin{aligned}
s_{c}(\tau, t)= & C \cdot s_{r}\left(\tau-\tau_{0}\right) \\
& \cdot s_{a}\left(t-t_{0}-\frac{c}{2} \frac{\tan \beta\left(r_{0}\right)}{v} \cdot\left(\tau-\tau_{0}\right)\right) \\
& \cdot \exp \left[-j \frac{4 \pi}{\lambda} r_{0} \cos \beta_{g}\right] \cdot \exp \left[j 2 \pi f_{c}\left(t-t_{0}\right)\right] \\
& \cdot \exp \left[-j 2 \pi f_{0} \cdot\left(\cos \beta_{g}-\cos \beta_{e f}\left(r_{0}\right)\right) \cdot\left(\tau-\tau_{0}\right)\right]
\end{aligned}
$$

By inspection of (17), it is possible to conclude that the phase ramp in range, given by the last exponential function, can be cancelled choosing the correct $\beta_{g}$ factor for a specific squint angle $\beta_{e f}\left(r_{0}\right)$. It is important to note that, as a result of the modification of the filter, the phase that contains the information of the range distance (first exponential) is also changed, being multiplied by the new factor. This implies that the new interferometric phase, if we follow the same process in the second image, is now expressed by

$$
\phi_{\mathrm{int}}=-\frac{4 \pi}{\lambda}\left(r_{1}-r_{2}\right) \cdot \cos \beta_{g}
$$

Nevertheless, this factor can be taken into account during the subsequent step of geocoding, so this scaling of the interferometric phase of the whole scene should not be considered harmful because it can be compensated for.

Since the range phase ramp has been eliminated for a specific squint, it implies that the phase errors coming from misregistration have been removed for this specific squint value. However, in a general case, the effective squint angle $\beta_{\text {ef }}$ could be characterized by an important variability along the range dimension. Indeed, if a range-varying factor $\beta_{g}$ was applied in (16), the interferometric phase value would take the form

$$
\phi_{\mathrm{int}}=-\frac{4 \pi}{\lambda}\left(r_{1} \cdot \cos \beta_{g}\left(r_{1}\right)-r_{2} \cdot \cos \beta_{g}\left(r_{2}\right)\right)
$$



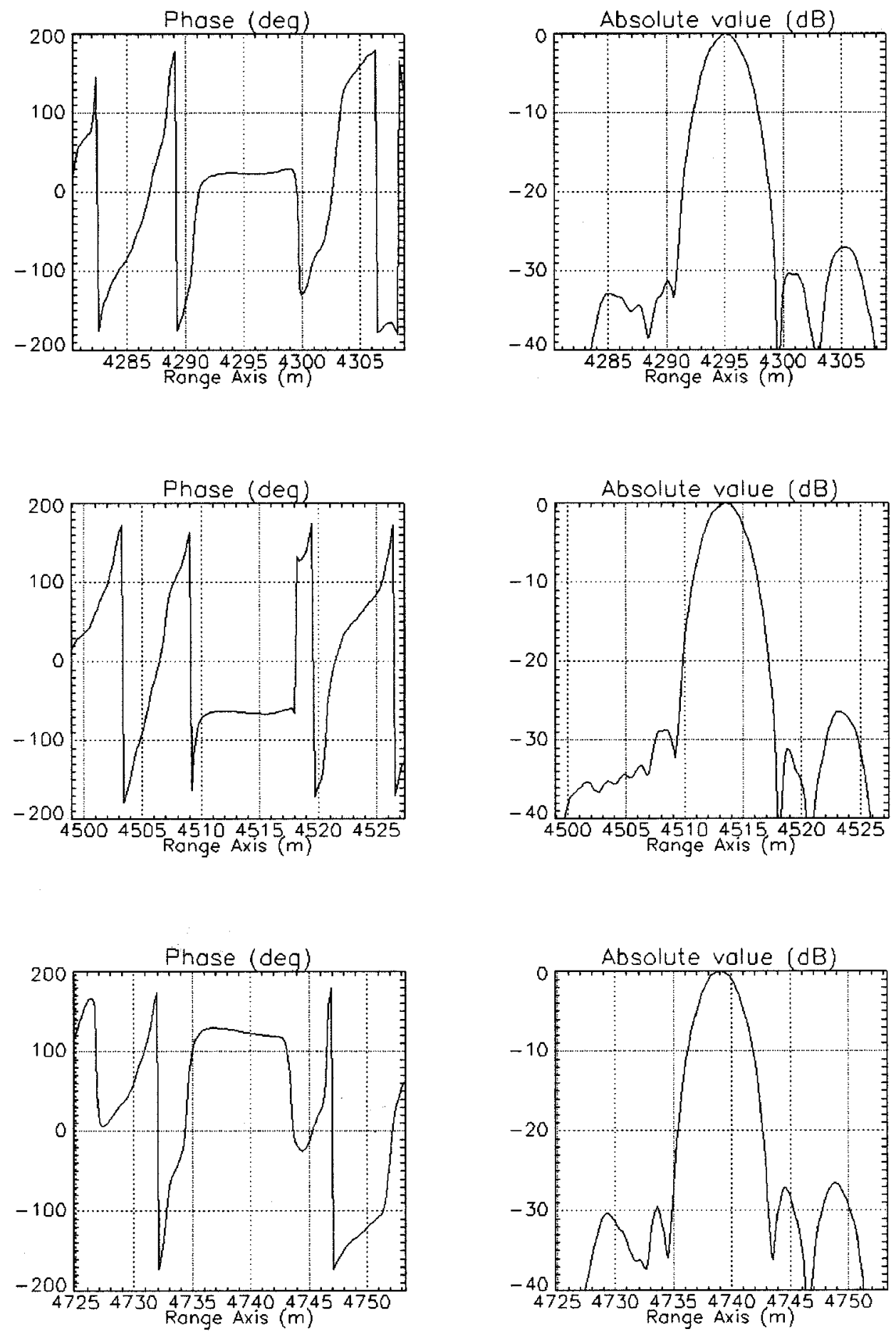

Fig. 5. IRF in range for the five corner reflectors focused with the modified azimuth compression filter function. The near, middle, and far range corner reflectors from Fig. 1 have been selected for representation.

introducing in this way an unknown phase shift that could not be recovered during geocoding, where the distance $r_{2}$ has to be found from $\phi_{\text {int }}[$ see (20)].

This filter has been applied to the X-Band E-SAR data and the result on the range IRFs of the corner reflectors is displayed in Fig. 5. In this case, the applied value of $\beta_{g}$ is $2.3^{\circ}$ (combination of real squint and MoCo term) and constant since the squint was considered to be the same for all range positions. An important reduction of the range phase ramp for all the targets could be obtained. The residual ramps are due to the range varying nature of the motion compensation, which could not be accounted for precisely for all corner reflector positions.

\section{B. Precise Geocoding Based on Estimated Misregistration}

The limitations of the previous method are twofold. First, the filter cannot be updated for each range position as explained by (19). Second, it is not possible to choose the best filter for all azimuth positions. This is due to the fact that the modified filter 
is applied in the azimuth spectral domain, but the MoCo term is variable also in azimuth.

Another method to compensate the phase bias is described in this section, trying to avoid the previous limitations. The concept is different from modifying any filter of the processing chain. This technique is directly based on the values of the calculated interferogram and therefore, it is suitable for integration into the geocoding part of the processing without changing the processor phase functions. Starting from the unwrapped interferometric phase, we will show that it is possible to estimate with a certain degree of precision the actual misregistration between both images and, in this way, remove the phase bias relying on the expressions obtained in the Sections II and III.

The process of relating the measured interferometric phase to a position and height on the Earth's surface is generally called geocoding [28], [29]. One of the first steps of this process consists of computing the distance $r_{2}$ between the target and the second antenna (slave antenna) of the system, starting from the interferometric phase value $\phi$ unwrapped [12] and with the flatEarth term

$$
r_{2}=r_{1}+\frac{\lambda \phi}{4 \pi}
$$

With the pair of values $r_{1}, r_{2}$, the radar/Earth geometry must be solved in order to find the position of the desired point (see Fig. $6)$. The problem is that any possible error in the interferometric phase is propagated to the $r_{2}$ distance by means of (20). The computed value of $r_{2}$ is degraded as long as a phase bias exists, and then the final position and height of the geocoded point can contain an error of several meters in the squinted case (see Section III-A ).

However, with this erroneous estimation of $r_{2}$, it is possible to obtain an initial guess for the position of this point in the second image, after the coregistration process. The technique to be presented here is based on the estimation of the misregistration. The main point is to know the value of

$$
\Delta r_{m i s}=r_{1}-r_{2, a c}
$$

where the subindex $a c$ stands for "after coregistration." The variable $\Delta r_{m i s}$, which ideally should be zero, is the actual distance between the position of both impulse responses when the interferometric channels are combined. On the other hand, the coregistration function $f_{\text {coreg }}$ that has been applied before the interferogram formation must be considered perfectly known

$$
r_{2, a c}=f_{c o r e g}\left(r_{2}\right)
$$

As an example, for the scaling properties of the ECS algorithm, the coregistration function is expressed by

$$
f_{\text {coreg }}(r)=\alpha \cdot\left(r-r_{r e f}\right)+r_{r e f 2}
$$

where $\alpha$ and $r_{r e f}$ are the scaling parameters of the ECS algorithm, and $r_{r e f 2}$ is slightly different from $r_{r e f}$ because it contains the term which accounts for the coarse coregistration.

The important idea of this method is that it is possible to substitute the first position of $r_{2}$ into (21) and (22) to get an estimate

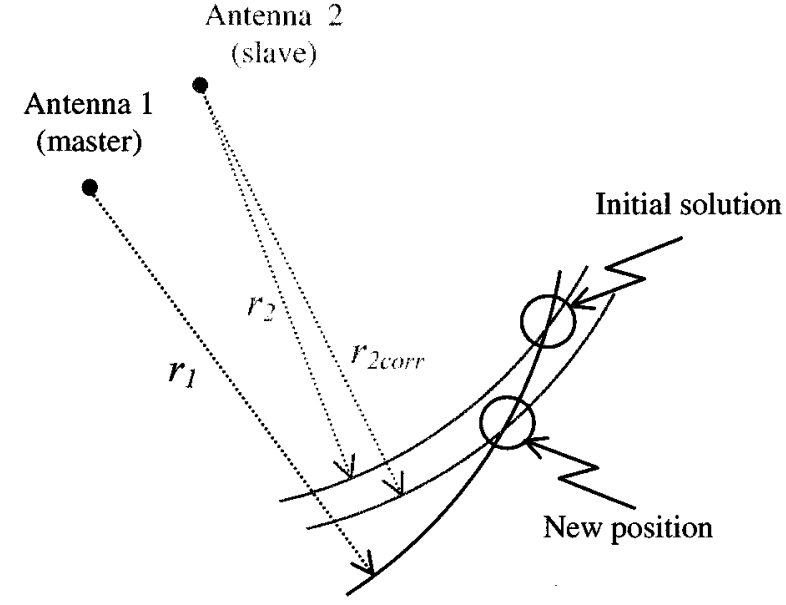

Fig. 6. Interferometric geometry for improved geocoding with the iterative approach.

of the residual misregistration. Hence, we can compute the interferometric phase bias based on its relation with misregistration, given by (15). Obviously, the knowledge of the phase bias is extremely useful, since it gives a much more accurate estimation of the correct interferometric phase. Actually, this process can be implemented directly on $r_{2}$, since its relation with the phase depends only on the well known factor $4 \pi / \lambda$. In this way, the corrected value for this distance is

$$
r_{2, \text { corr }}=r_{2}-\Delta r_{\text {mis }} \cdot\left(1-\cos \beta_{e f}\left(r_{2}\right)\right) \text {. }
$$

If the misregistration estimation is accurate, then the new value of $r_{2}$ is also more precise and consequently, the final geocoded position is improved (see Fig. 6). Indeed, it is possible to check if the modification is correct by applying again (21) and (22) to the new distance. Then, the process becomes iterative until the values of the misregistration and the phase bias agree with each other

$$
r_{2, n+1}=r_{2,0}-\left\{r_{1}-f_{c o r e g}\left(r_{2, n}\right)\right\} \cdot\left(1-\cos \beta_{e f}\left(r_{2, n}\right)\right)
$$

where $n$ is the iteration number. The iteration would be finished when the second term of (25) is lower than a certain threshold. A block diagram of the proposed technique is depicted in Fig. 7. The operations to be performed within the first iteration step are presented on the left hand side, while the complete iterative scheme is detailed on the right. This correction, indeed, could be applied also in conjunction with the filter of (16). In that case, (24) and (25) should take the new factor $\beta_{g}$ into account, as corresponds to (17).

\section{Iterative Method: Simulation Results}

In order to test the performance of the iterative method of the previous section, E-SAR data have been simulated with the system parameters of Table I. The purpose is to have available a set of data with much higher squint values, i.e., with an important spectral shift and corresponding interferometric phase bias. The simulation has been performed with a high variability of the squint angle from near to far range (from 7.8-11.3'). The deviations from the nominal track have not been included and 

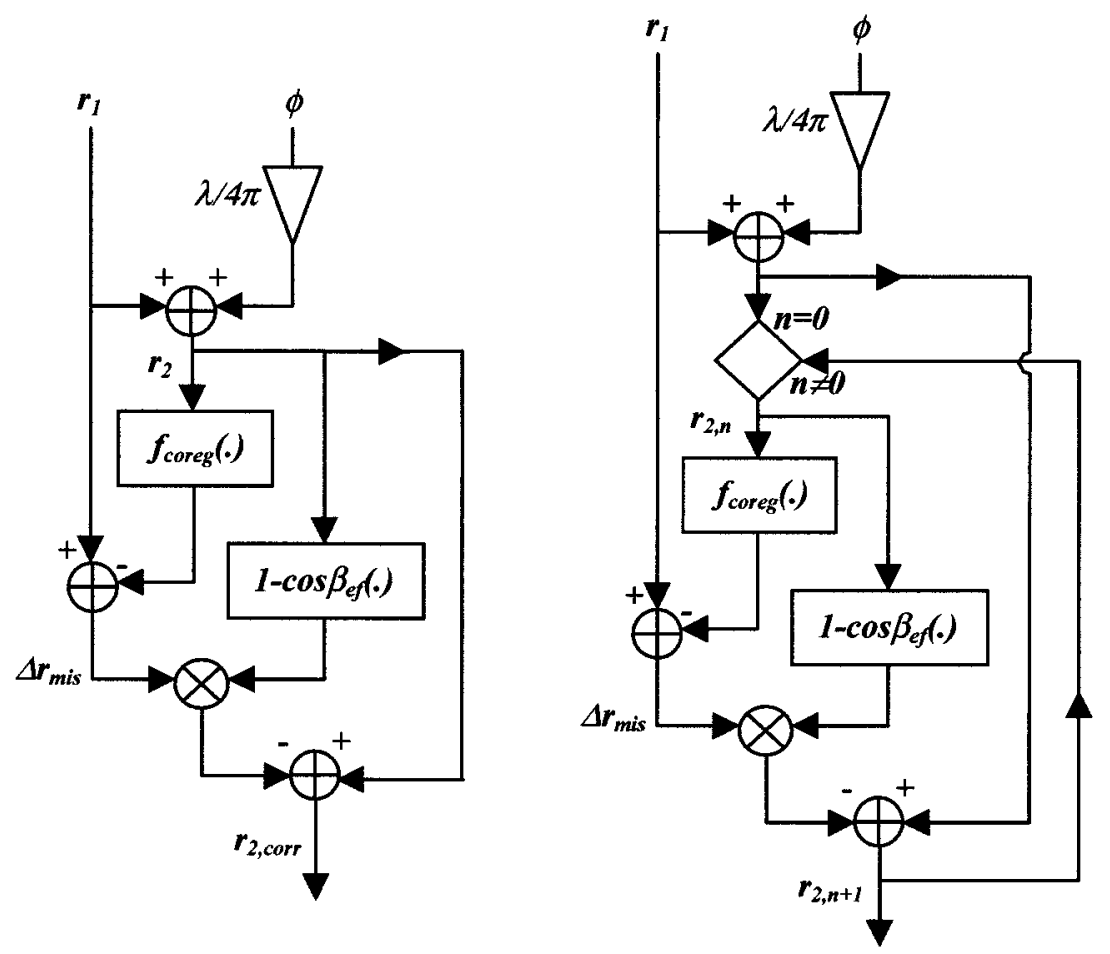

Fig. 7. Block diagram of the correction procedure. Left side: one iteration. Right side: $n$ iterations.

TABLE II

MisRegistration AND Phase Bias for Simulated Point TARgets

\begin{tabular}{c|c|c|c}
\hline Target & Range mis-registration (m) & $\begin{array}{c}\text { Theoretical Phase Bias } \\
\text { (deg) }\end{array}$ & $\begin{array}{c}\text { Detected Phase Bias } \\
\text { from Simulation (deg) }\end{array}$ \\
\hline P0 & $-0.060 \mathrm{~m}$ & -13.36 & -12.39 \\
PI & $-0.118 \mathrm{~m}$ & -38.86 & -39.64 \\
P2 & $-0.088 \mathrm{~m}$ & -35.18 & -32.49 \\
\hline
\end{tabular}

therefore, there is no contribution of the MoCo term to the phase ramp in this case. The data contain point targets in near, mid and far range, located in the same zero-Doppler azimuth position.

The interferogram has been formed after a coregistration process based on the range scaling properties of the ECS algorithm and therefore, the function to be considered during the iterative method is the one expressed in (23). The simulated point targets are located at a local height of about $600 \mathrm{~m}$, whereas the scaling parameters, $\alpha$ and $r_{r e f}$, for the coregistration step were calculated for a terrain height of $400 \mathrm{~m}$, in order to simulate the effects of the unknown elevation of the observed area. The misregistration in every point target is displayed in Table II, as well as the phase bias detected in the simulation in comparison to the expected theoretical one. As we can see, the detected bias reaches high values $\left(40^{\circ}\right)$, which would correspond to a maximum height error of approximately $4 \mathrm{~m}$.

After employing the proposed method with three iterations, we have obtained the phase bias estimations shown in Fig. 8 expressed in degrees. As we can see, they are in agreement with the theoretical ones predicted by the equations derived in this paper (Table II). In this way, the estimated phase error can be subtracted yielding a high reduction of the phase bias for all the point targets within the swath (Fig. 9). The resulting height errors are in the order of $20 \mathrm{~cm}$ in the worst case (point $P 2$ ). The result of the iteration gives a very precise estimation of the errors generated by the combination of squint and misregistration.

As can be noticed in Fig. 8, the method converges very fast; in this case one iteration would be enough. The reason can be found in the fact that a linear function for coregistration (23) has been used with coefficients that assure the convergence. In Appendix $\mathrm{C}$, the analysis of this aspect is derived.

\section{Iterative Method: Results with Airborne Data}

The validity of the iterative approach has also been checked by means of the E-SAR airborne data (Table I). For these data, the targets are characterized by a noticeable phase ramp in range, as shown in Fig. 3. This phase evolution, in combination with a specific misregistration, leads to the interferometric phase bias mentioned above. In this case, the reflectors are located in a nearby position in range, so in order to make evident the different bias in each of them we have imposed a different misregistration from $P 0-P 4$ (see Table III). (The reflectors have been called $P i$ from near to far range, with 


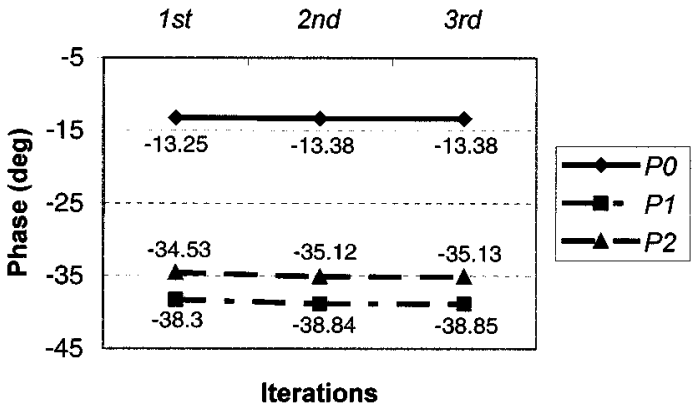

Fig. 8. Interferometric phase bias estimated with the iterative method for the simulated point targets (three iterations).

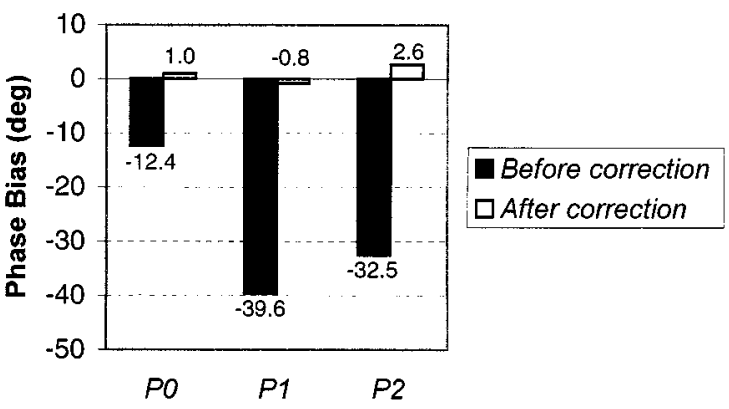

Fig. 9. Interferometric phase improvement obtained with the iterative method for the simulated point targets.

$i=0, \cdots, 4$.). As we see, the misregistration in $P 0$ and $P 4$ reaches the value of approximately $25 \%$ of the resolution cell $(3 \mathrm{~m})$. In the same table, we also represent the values of the phase ramp for every point (2nd column) and, from them, the expected bias predicted by the theoretical formulation of (27) (3rd column).

It is important to note that, in order to carry out an accurate study of only this source of error, neglecting the other possible contributions, it is necessary to compare the results to the ones obtained in the perfect coregistration case. With this purpose, another interferogram with exact coregistration for all point targets has been formed. After the detection of the phase values for every reflector, the biased values shown in Table III (4th column) have been found. They are in good agreement with the expected ones. The next step is to use the iterative approach in order to correct the phase bias. Also in this case it has been found that one iteration is enough for reaching a good estimate of the theoretical bias. After subtracting the estimations from the actual bias, the accuracy shown in Fig. 10 has been obtained. As one can see, the bias in all the control points has drastically been reduced, from $-13.68^{\circ}$ to $-1.14^{\circ}$ in the worst case.

The performance of the method, which is applied pixel by pixel, is also accurate in areas with no strong point-like corner reflectors, since it is based on the phase itself and not on the reflectivity. For those pixels where the interferometric phase contains a high level of noise, the importance of this correction decreases since the squint effect represents a minor impact in comparison to the high decorrelation. Thus, it would be reasonable to mask these pixels in order not to apply the correction. In this test with real data, the presence of noisy values in shadowed
TABLE III

Misregistration, Phase RAmp, Expected Bias, and Detected BIAS FOR EVERY GROUND CONTROL POINT

\begin{tabular}{l|c|c|c|c}
\hline Target & Mis-registration $(\boldsymbol{m})$ & $\begin{array}{c}\text { Phase Ramp (deg/m) } \\
\text { (Squint and Zd Order } \\
\text { MOCo) }\end{array}$ & $\begin{array}{c}\text { Expected Bias } \\
(\text { deg })\end{array}$ & $\begin{array}{c}\text { Detected Bias } \\
(\text { deg })\end{array}$ \\
\hline P0 & -0.692 & 18.04 & -12.48 & -13.68 \\
P1 & -0.348 & 18.76 & -6.53 & -7.11 \\
P2 & 0 & 19.39 & 0 & -0.17 \\
P3 & 0.357 & 19.94 & 7.12 & 7.00 \\
P4 & 0.722 & 20.44 & 14.76 & 14.63 \\
\hline
\end{tabular}

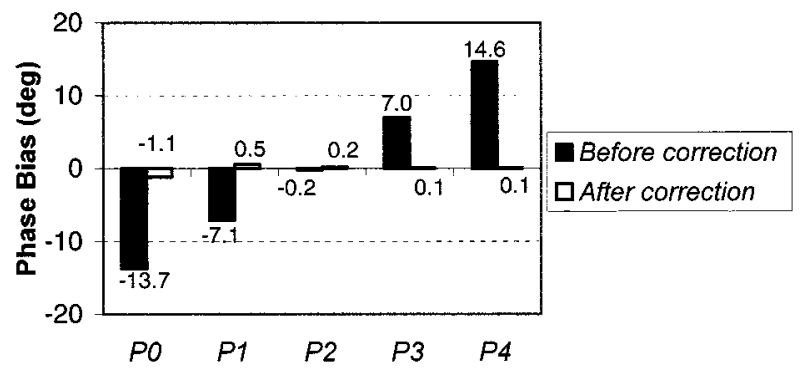

Fig. 10. Interferometric phase improvement obtained with the iterative method for the five corner reflectors.

cells has not affected the convergence. The theoretical explanation is shown in Appendix C.

As a conclusion to sections $\mathrm{C}$ and $\mathrm{D}$, we point out that the theoretical method based on the estimation of the misregistration has successfully shown its validity to improve the results not only in a simulated scenario but also with data coming from an operative system like E-SAR.

\section{CONCLuSIONS}

In this paper a complete SAR signal analysis from the interferometric point of view has been developed. The formulation includes the effect of the squint angle in the SAR impulse response function, as well as other terms coming from the motion compensation step during processing of airborne data. The presence of a phase ramp in the range direction has been pointed out, as well as its consequences on the interferometric technique for air- and spaceborne SAR platforms. In particular, it has been shown that a noticeable phase bias exists in the presence of misalignment between both images in the squinted case. This bias reduces the capability of the interferometric technique to retrieve the correct topographic information, especially for airborne sensors.

In this work, two new methods for the reduction of this kind of limitation have been presented theoretically and evaluated by means of simulations and real data from the E-SAR system. They overcome the problems related to the squinted geometry and reduce the strong requirements on range coregistration accuracy. Actually, the accuracy of range coregistration should increase proportionally to the squint angle, which is not feasible from a practical point of view. Indeed, perfect coregistration in range will never be possible prior to the geocoding step in case no external DEM is available. Therefore, the two methods for phase bias compensation presented in this paper are of extreme 
importance for improving the accuracy of DEM generated by airborne SAR platforms in the presence of squint.

\section{APPENDIX A}

\section{TAYLOR EXPANSION OF AZIMUTH-COMPRESSED PHASE}

If the azimuth-compressed signal [after the application of (5)] is evaluated for a range time close to the IRF maximum, we get the following:

$$
\begin{aligned}
S_{c}\left(\tau=\tau_{0}+\Delta \tau, f_{a}\right) \\
=C_{0} \cdot s_{r}(\Delta \tau) \cdot w_{a}\left(\frac{f_{a}-f_{c}}{f_{r}}\right) \cdot \exp \left[-j 2 \pi f_{a} t_{0}\right] \\
\quad \cdot \exp \left[j \frac{4 \pi}{\lambda} \Delta r \sqrt{1-\left(\frac{\lambda f_{a}}{2 v}\right)^{2}}\right] \cdot \exp \left[-j \frac{4 \pi}{\lambda}\left(r_{0}+\Delta r\right)\right]
\end{aligned}
$$

where $\Delta \tau=2 \cdot(\Delta r / c)$. In the squinted case, the Taylor expansion of the square root of (A1) must be calculated around the Doppler centroid frequency. In this way, the filtered response becomes

$$
\begin{array}{r}
S_{c}\left(\tau=\tau_{0}+\Delta \tau, f_{a}\right) \approx C_{0} \cdot s_{r}(\Delta \tau) \cdot w_{a}\left(\frac{f_{a}-f_{c}}{f_{r}}\right) \\
\cdot \exp \left[-j 2 \pi f_{a} t_{0}\right] \cdot \exp [j \Phi]
\end{array}
$$

where the phase $\Phi$ can be expressed by a second order polynomial

$$
\Phi=\phi_{0}+\phi_{1}\left(f_{a}-f_{c}\right)+\phi_{2}\left(f_{a}-f_{c}\right)^{2}
$$

and the three coefficients are

$$
\begin{aligned}
& \phi_{0}=-\frac{4 \pi}{\lambda} r_{0}-\frac{4 \pi}{\lambda} \Delta r\left[1-\sqrt{1-\left(\frac{\lambda f_{c}}{2 v}\right)^{2}}\right] \\
& \phi_{1}=-\frac{4 \pi}{\lambda} \Delta r \frac{\left(\frac{\lambda}{2 v}\right)^{2} f_{c}}{\sqrt{1-\left(\frac{\lambda f_{c}}{2 v}\right)^{2}}} \\
& \phi_{2}=-\frac{2 \pi}{\lambda} \Delta r \frac{\left(\frac{\lambda}{2 v}\right)^{2}}{\left[1-\left(\frac{\lambda f_{c}}{2 v}\right)^{2}\right]^{3 / 2}} .
\end{aligned}
$$

With respect to the constant term, it shows that the presence of the squint induces a change on the phase of the response in a point located at a distance $\Delta r$ from the peak

$$
\phi_{0}=-\frac{4 \pi}{\lambda}\left(r_{0}+\Delta r \cdot(1-\cos \beta)\right)
$$

Indeed, (A5) describes a linear behavior with respect to $\Delta r$, which induces a linear phase ramp in range. On the other hand, the first order coefficient in (A4) can be rewritten as

$$
\phi_{1}=-2 \pi \frac{\tan \beta}{v} \Delta r
$$

This factor leads to a shift in azimuth time when transforming the signal back to the time domain. Specifically, it is responsible for the shift of the sidelobes that can be observed in the zero-Doppler image geometry. Finally, the second order term imposes a quadratic phase evolution, which is

$$
\phi_{2}=-\frac{2 \pi}{\lambda} \Delta r \cdot\left(\frac{\lambda}{2 v}\right)^{2} \frac{1}{\cos ^{3} \beta} .
$$

Equation (A7) indicates a slight azimuth defocusing for those parts of the signal not located at the range IRF maximum, meaning that the range sidelobes are not properly compressed. Nevertheless, it can be shown that for all known air- and spaceborne SAR sensors, this quadratic phase factor is negligible for small $\Delta r$ (main lobe).

\section{APPENDIX B \\ EFFECTS ON COHERENCE}

In Section III, it has been shown that the squint angle, combined with misregistration, introduces a range dependent phase bias of the interferometric phase coming from a point target. However, in a real case, most of the natural surfaces that can be observed by a SAR sensor are formed by resolution cells with a continuous pattern of scatterers, distributed in a specific way on the ground. That is the reason why the phase for every point in an interferogram has been traditionally considered a random variable, with specific mean and absolute deviation values [21], [30]. Hence, it is possible to derive the concept of coherence and phase bias.

In order to extend the results obtained with point scatterers by means of it, one can only assess the effects on the phase bias. It is useful to compute the statistics of a more complex resolution cell, obtaining in this way the changes in the interferometric coherence.

The geometry for the calculation of the cell statistics is shown in Fig. 11. It is considered that both antennas receive the scattered field from the same resolution cell, which consists of uniformly distributed and independent scatterers. Under these assumptions, the cross correlation between the received fields for that specific pixel is traditionally considered to be [24]

$$
\begin{aligned}
\left\langle s_{1} \cdot s_{2}^{*}\right\rangle= & C_{1} \cdot C_{2} \cdot \exp \left[-j \frac{4 \pi}{\lambda}\left(r_{1}-r_{2}\right)\right] \cdot \int \sigma_{0} \\
& \cdot \exp \left[-j \frac{4 \pi}{\lambda}\left(\sin \theta_{1}-\sin \theta_{2}\right)\left(y-y_{0}\right)\right] \\
& \cdot \operatorname{sinc}\left(\left(y-y_{0}\right) / R_{y 1}\right) \cdot \operatorname{sinc}\left(\left(y-y_{0}\right) / R_{y 2}\right) \cdot d y
\end{aligned}
$$

where

〈. expectation operator; 


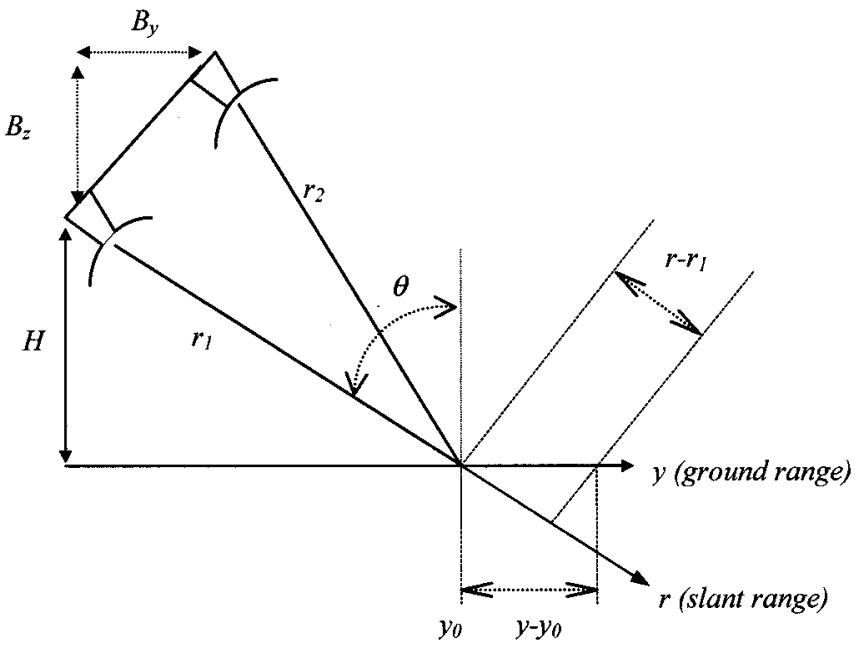

Fig. 11. Geometry for the calculation of the interferometric statistics.

$$
\begin{array}{ll}
R_{y 1} \text { and } R_{y 2} & \begin{array}{l}
\text { resolutions in ground range for each image; } \\
\text { mean absolute deviation of the scatterers re- } \\
\sigma_{0}
\end{array} \\
& \begin{array}{l}
\text { flectivity function } \psi\left(\sigma_{0}=\left\langle\psi(y)^{2}\right\rangle\right) \\
C_{1} \text { and } C_{2}
\end{array} \\
\text { arbitrary complex constants. }
\end{array}
$$

However, our goal is to study the effects of the phase ramp in range, so (B1) must be modified in order to include the correct expression of both impulse response functions in this case

$$
\begin{aligned}
s_{i}(\tau)=C_{i} \cdot \int \psi(y) \cdot \operatorname{sinc}\left(\tau-\frac{2}{c}\left(r_{i}+\Delta y \cdot \sin \theta\right)\right) \\
\cdot \exp \left[-j \frac{4 \pi}{\lambda}\left(r_{i}+\Delta y \cdot \sin \theta\right)\right] \\
\cdot \exp \left[-j \frac{4 \pi}{\lambda}\left(1-\cos \beta_{e f}\right)\right. \\
\left.\cdot\left(\frac{c \tau}{2}-\left(r_{i}+\Delta y \cdot \sin \theta\right)\right)\right] \cdot d y
\end{aligned}
$$

where $\psi$ is the reflectivity function in ground range. The last exponential term is introduced to account for the phase ramp. Therefore, the cross correlation results in

$$
\begin{aligned}
\left\langle s\left(\tau_{1}\right) \cdot s\left(\tau_{2}\right)^{*}\right\rangle & =C_{1} \cdot C_{2} \cdot \exp \left[-j \frac{4 \pi}{\lambda}\left(r_{1}-r_{2}\right)\right] \cdot \int \sigma_{0} \\
& \cdot \exp \left[-j \frac{4 \pi}{\lambda}\left(\sin \theta_{1}-\sin \theta_{2}\right)\left(y-y_{0}\right) \cos \beta_{e f}\right] \\
& \cdot \operatorname{sinc}\left(\left(y-y_{0}\right) / R_{y 1}\right) \cdot \operatorname{sinc}\left(\left(y-y_{0}\right) / R_{y 2}\right) \cdot d y
\end{aligned}
$$

where $\tau_{i}$ is the time measured at the center of the cell $\left(\tau_{i}=\right.$ $2 r_{i} / c$ ). The only difference from (B1) is the existence of the effective squint angle in the exponential term. It is important to note that we are assuming that the squint angle is the same for both acquisitions, so there is no decorrelation due to different Doppler centroids [31]. That leads to a small change in the coherence of the resolution cell, which can be calculated after normalization of (B3)

$$
\gamma=1-\frac{f_{0} \cdot\left|\sin \theta_{1}-\sin \theta_{2}\right|}{W \cdot \sin \theta} \cdot \cos \beta_{e f}
$$

where

$\theta_{i} \quad$ look angle for the antenna position $i$;

$\theta \quad$ average;

$W$ available bandwidth.

Expression (B4) considers the well-known spatial or baseline decorrelation [23], [32], [33], which appears due to the different point of view in the two acquisitions, but in this case the factors coming from the exact impulse response expression have been included.

The next objective is to find out the consequences of a specific misregistration. It is possible if we take into account a possible error $\tau_{m i s}$ in the position of one of the two fields

$$
\begin{aligned}
& \left\langle s\left(\tau_{1}\right) \cdot s\left(\tau_{2}-\tau_{m i s}\right)^{*}\right\rangle \\
& =C_{1} \cdot C_{2} \cdot \exp \left[-j \frac{4 \pi}{\lambda}\left(r_{1}-r_{2}\right)\right] \cdot \int \sigma 0 \\
& \quad \cdot \exp \left[-j \frac{4 \pi}{\lambda}\left(\sin \theta_{1}-\sin \theta_{2}\right)\left(y-y_{0}\right) \cos \beta_{e f}\right] \\
& \quad \cdot \exp \left[j \frac{4 \pi}{\lambda}\left(1-\cos \beta_{e f}\right) y_{m i s} \sin \theta_{2}\right] \\
& \quad \cdot \operatorname{sinc}\left(\left(y-y_{0}\right) / R_{y 1}\right) \cdot \operatorname{sinc}\left(\left(y-y_{0}-y_{m i s}\right) / R_{y 2}\right) \cdot d y
\end{aligned}
$$

where $y_{\text {mis }}$ is the displacement in ground range. By means of (B5), and defining the misregistration in slant range as

$$
\Delta r_{m i s}=y_{m i s} \cdot \sin \theta_{2}
$$

it is shown that the last exponential term of (B5) introduces the following bias to the interferometric phase:

$$
\phi_{\text {bias }}=\frac{4 \pi}{\lambda} \Delta r_{m i s} \cdot\left(1-\cos \beta_{e f}\right)
$$

which is the same as the one found in the point targets case. On the other hand, by developing (B5), it is possible to show that the misregistration introduces a degradation of the coherence, as it has been demonstrated in other works [22], [25].

\section{APPENDIX C \\ CONVERGENCE OF ITERATIVE METHOD}

The iterative formula described by (25) can be written as

$$
r_{2, n+1}=r_{2,0}-\Delta r_{2, n}
$$

where $\Delta r_{2, n}$ is defined as the applied correction, with $n$ ranging from $0-\infty$. Its value is expressed in terms of the previous iteration as

$\Delta r_{2, n+1}=\left\{r_{1}-f_{\text {coreg }}\left(r_{2,0}-\Delta r_{2, n}\right)\right\} \cdot\left(1-\cos \beta_{e f}\left(r_{2,0}\right)\right)$

where we have considered that the variation of $\beta_{e f}$ in the range of the corrections $\Delta r_{2, n}$ is negligible since they are in the order of $\mathrm{cm}$ even in the presence of noise. Therefore, the series convergence depends directly on the shape of the function $f_{\text {coreg }}$. 
In the case of a linear coregistration, as the one employed by the ECS algorithm (23), (C2) converges to

$$
\Delta r_{2, \infty}=\frac{\Delta r_{2,0}}{1-\alpha \cdot\left(1-\cos \beta_{e f}\left(r_{2,0}\right)\right)}
$$

as long as the following condition is fulfilled:

$$
\left|\alpha \cdot\left(1-\cos \beta_{e f}\left(r_{2,0}\right)\right)\right|<1 \text {. }
$$

In this particular case, the final value of $r_{2}$ can be written as a function of the initial interferometric phase from (C3) and (20)

$r_{2, \infty}=\frac{r_{1} \cos \beta+\frac{\lambda \phi}{4 \pi}-\left(\alpha \cdot r_{r e f}-r_{r e f 2}\right) \cdot\left(1-\cos \beta_{e f}\left(r_{2,0}\right)\right)}{1-\alpha \cdot\left(1-\cos \beta_{e f}\left(r_{2,0}\right)\right)}$

In (C5), it is clear that the effect of phase noise is translated proportionally into the corrected value of $r_{2}$, but without affecting the convergence conditions. Equation (C5) has shown to be valid for the example with real data of Section III, where the condition given by (C4) is fulfilled. Moreover, (C3) shows that, for the specific values of $\alpha$ and $\beta_{e f}$ of that case, the subsequent iterations after the first one provide no significant improvement. In a general case, where $f_{\text {coreg }}$ can have an arbitrary evolution over range, $(\mathrm{C} 2)$ should be studied specifically to determine the requirements of convergence.

\section{ACKNOWLEDGMENT}

The authors would like to thank the anonymous reviewers for their comments and suggestions.

\section{REFERENCES}

[1] J. C. Curlander and R. N. McDonough, Synthetic Aperture Radar: Systems and Signal Processing. New York: Wiley, 1991.

[2] C. Wu, K. Y. Liu, and M. Jin, "Modeling and a correlation algorithm for spaceborne SAR signals," IEEE Trans. Geosci. Remote Sensing, vol. GE-18, pp. 563-574, Sept. 1980.

[3] R. Bamler, "A comparison of range-Doppler and wavenumber domain SAR focusing algorithms," IEEE Trans. Geosci. Remote Sensing, vol. 30, pp. 706-713, July 1992

[4] C. Cafforio, C. Prati, and F. Rocca, "SAR data focusing using seismic migration techniques," IEEE Trans. Aerosp. Electron. Syst., vol. 27, pp. 199-207, Mar. 1991.

[5] R. K. Raney, H. Runge, R. Bamler, I. G. Cumming, and F. H. Wong, "Precision SAR processing using chirp scaling," IEEE Trans. Geosci. Remote Sensing, vol. 32, pp. 786-799, July 1994.

[6] M. Y. Jin and C. Wu, "A SAR correlation algorithm which accomodates large range migration," IEEE Trans. Geosci. Remote Sensing, vol. 22 pp. 592-597, Sept. 1984.

[7] D. Just and B. Scättler, "Doppler characteristics of the ERS-1 yaw steering mode," in Proc. IGARSS, Houston, TX, 1992.

[8] R. K. Raney, A. P. Luscombe, E. J. Langham, and S. Ah-med, "RADARSAT," Proc. IEEE, vol. 79, pp. 839-849, 1991.

[9] G. W. Davidson and I. G. Cumming, "Signal properties of spaceborne squint-mode SAR,” IEEE Trans. Geosci. Remote Sensing, vol. 35, pp. 611-617, May 1997.

[10] G. W. Davidson, I. G. Cumming, and M. R. Ito, "A chirp scaling approach for processing squint mode SAR data," IEEE Trans. Aerosp. Electron. Syst., vol. 32, pp. 121-133, Jan. 1996.

[11] L. C. Graham, "Synthetic interferometer radar for topographic mapping," Proc. IEEE, vol. 62, pp. 763-768, June 1974.

[12] R. M. Goldstein, H. A. Zebker, and C. L. Werner, "Satellite radar interferometry: Two-dimensional phase unwrapping," Radio Sci., vol. 23, pp. 713-720, July 1988
[13] R. Scheiber and A. Moreira, "Improving co-registration accuracy of interferometric SAR images using spectral diversity," in Proc. IGARSS, Hamburg, Germany, July 1999.

[14] R. Horn, "The DLR airborne SAR project E-SAR," in Proc. IGARSS, May 1996, pp. 1624-1628.

[15] A. Moreira, J. Mittermayer, and R. Scheiber, "Extended chirp scaling algorithm for air- and spaceborne SAR data processing in stripmap and ScanSAR imaging modes," IEEE Trans. Geosci. Remote Sensing, vol. 34, pp. 1123-1136, Sept. 1996.

[16] R. K. Raney, "Considerations for SAR image quantification unique to orbital systems," IEEE Trans. Geosci. Remote Sensing, vol. 29, pp. 754-760, Sept. 1991.

[17] A. Moreira and Y. Huang, "Airborne SAR processing of highly squinted data using a chirp scaling approach with integrated motion compensation," IEEE Trans. Geosci. Remote Sensing, vol. 30, pp. 706-713, Sept. 1994.

[18] J. C. Kirk, "Motion compensation for synthetic aperture radar," IEEE Trans. Aerosp. Electron. Syst., vol. AESP-11, pp. 338-348, May 1975.

[19] D. Blacknell, A. Freeman, S. Quegan, I. A. Ward, I. P. Finley, C. J. Oliver, R. G. White, and J. W. Wood, "Geometric accuracy in airborne SAR images," IEEE Trans. Aerosp. Electron. Syst., vol. 25, pp. 241-256, Mar. 1989.

[20] D. Stevens, I. Cumming, and A. Gray, "Options for airborne interferometric SAR motion compensation," IEEE Trans. Geosci. Remote Sensing, vol. 33, pp. 409-419, Mar. 1995.

[21] S. N. Madsen, "Topographic mapping using radar interferometry: Processing techniques," IEEE Trans. Geosci. Remote Sensing, vol. 31, pp. 246-255, Jan. 1993.

[22] R. Bamler and D. Just, "Phase statistics and decorrelation in SAR interferograms," in Proc. IGARSS, 1993, pp. 980-984.

[23] E. Rodriguez and J. M. Martin, "Theory and design of interferometric synthetic aperture radars," Proc. Inst. Elect. Eng. F, vol. 39, pp. 147-159, Apr. 1992.

[24] H. A. Zebker and J. Villasenor, "Decorrelation in interferometric radar echoes," IEEE Trans. Geosci. Remote Sensing, vol. 30, pp. 950-959, Sept. 1992.

[25] F. K. Li and R. M. Goldstein, "Studies of multibaseline spaceborne interferometric synthetic aperture radars," IEEE Trans. Geosci. Remote Sensing, vol. 28, pp. 88-97, Jan. 1990.

[26] H. Runge, "Benefits of antenna yaw steering for SAR," in Proc. IGARSS, Helsinki, Finland, 1991, pp. 257-259.

[27] D. Geudtner, P. W. Vachon, K. E. Mattar, and A. L. Gray, "RADARSAT repeat-pass interferometry," in Proc. IGARSS, Seattle, WA, 1998.

[28] G. Schreier, SAR Geocoding: Data and Systems. Karlsruhe, Germany: Wichmann, 1993.

[29] W. Goblirsch and P. Pasquali, "Algorithms for calculation of digital surface models from the unwrapped interferometric phase," in Proc. IGARSS, Lincoln, NE, 1996, pp. 656-658.

[30] J. Lee, K. W. Hoppel, S. A. Mango, and A. R. Miller, "Intensity and phase statistics of multilook polarimetric and interferometric SAR imagery," IEEE Trans. Geosci. Remote Sensing, vol. 32, pp. 1017-1028, Sept. 1994.

[31] M. Schwäbisch and D. Geudtner, "Improvement of phase and coherence map quality using azimuth prefiltering: Examples from ERS-1 and X-SAR," in Proc. IGARSS, Florence, Italy, 1995, pp. 205-207.

[32] F. Gatelli, A. M. Guarnieri, F. Parizzi, P. Pasquali, C. Prati, and F. Rocca, "The wavenumber shift in SAR interferometry," IEEE Trans. Geosci. Remote Sensing, vol. 32, pp. 855-864, Sept. 1994.

[33] C. Prati and F. Rocca, "Improving slant-range resolution with multiple SAR surveys," IEEE Trans. Geosci. Remote Sensing, vol. 29, pp. 135-144, Jan. 1993.

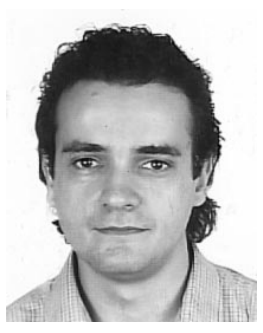

Marc Bara (S'99) was born in Barcelona, Spain, in 1972. He received the Diploma degree in electrical engineering in 1996 from the Polytechnic University of Catalonia (UPC), Barcelona, Spain, where he is currently pursuing the Ph.D degree in SAR processing and interferometry focused on motion compensation, geocoding, and design of interferometric SAR airborne systems.

Since 1996, he has been with the Electromagnetics and Photonics Engineering Group, Deparment of Signal Theory and Communications, UPC. 


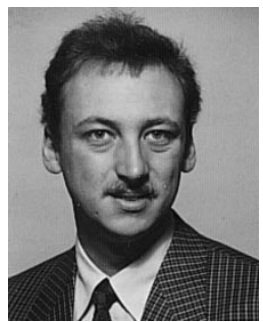

Rolf Scheiber received the Diploma degree in electrical engineering from the Technical University of München, München, Germany, in 1994, doing his thesis on the estimation of the Doppler parameters for the 2-D SAR processing

In 1994, he was with at the Institute for Communications Technology, German Aerospace Center (DLR), Oberpfaffenhofen, Germany, on channel measurements for mobile satellite communications. Since 1995, he has been with the Signal Processing Group, Institute for Radio Frequency Technology, DLR, as a Research Scientist. He developed an operational high precision interferometric SAR processing algorithm for airborne data and is presently responsible for the operational interferometric processing of the experimental SAR system of DLR (E-SAR).

Mr. Scheiber was awarded as a co-author with the 1996 GRSS Transactions Prize Paper Award for the contribution "Extended Chirp Scaling Algorithm for Air- and Spaceborne SAR Data Processing in Stripmap and ScanSAR Imaging Modes," in 1997. His current research interests include algorithm development for air- and spaceborne SAR interferometry, interferometric performance estimation, and algorithm development for real-time processing for future spaceborne SAR sensors.

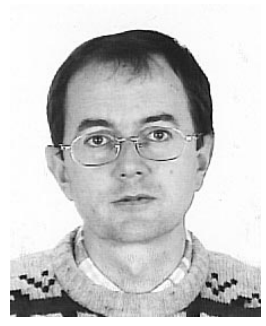

Antoni Broquetas (S'84-M'90) was born in Barcelona, Spain, in 1959. He received the Ing. degree in telecommunication engineering in 1985, and the Dr. Ing. degree in telecommunications engineering for his work on microwave tomography, in 1989, both from the Universitat Politécnica de Catalunya (UPC), Barcelona, Spain.

In 1986, he was a Research Assistant in the Portsmouth Polytechnic, Portsmouth, U.K., involved in propagation studies. In 1987, he joined the Department of Signal Theory and Communications, School of Telecommunicatoin Engineering, UPC, as a Professor. Currently, he is involved in research on radar imaging and remote sensing and is responsible of the research activities of the Institute of Geomatics.

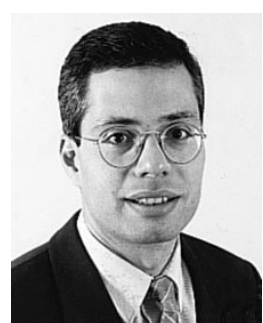

Alberto Moreira (M'92-S'96) was born in São José dos Campos, Brazil, in 1962. He received the B.S.E.E. and the M.S.E.E. degrees in 1984 and 1986, respectively, from the Aeronautical Technological Institute (ITA), Brazil, and the Eng. Dr. degree ("mit Auszeichung"-Hons.) from the Technical University of Münich, Münich, Germany, in 1993.

In 1987, he received a four-year scholarship from DAAD, Germany, to work toward the Ph.D. at the German Aerospace Center (DLR - Institut für Hochfrequenztechnik) in the area of SAR signal processing. From 1985 to 1986, he was with ITA as a Research Assistant and Consultant. From 1992 to 1995 , he was the Head of the Signal Processing Group with DLR and was responsible for the development of a high resolution real-time airborne SAR processor. As Chief Scientist and Engineer with DLR since 1996, he manages the DLR SAR Technology Department. Under his leadership, the DLR airborne SAR system has been upgraded to operate in innovative imaging modes like polarimetric SAR interferometry and polarimetric SAR tomography. He is currently serving as a member of the Administrative Committe of the Geoscience and Remote Sensing Society.

Dr. Moreira was the recipient of the DLR Science Award in 1995. In 1996, he and his colleagues received the IEEE 1996 Transactions Prize Paper Award for the development of the so-called "Extended Chirp Scaling Algorithm." In 1999, he received the IEEE AESS Fred Nathanson Memorial Award to the Young Radar Engineer of the Year. His professional interests and research areas encompass SAR end-to-end system design and analysis, SAR signal processing and interferometry, innovative radar techniques, and expert systems for data fusion and classification. 